\title{
Editorial
}

\section{PAG and the latest trends in breeding research}

The $20^{\text {th }}$ International Plant and Animal Genome Conference (PAG XX) was held in San Diego, USA on January 1418,2012 . Aimed at providing a forum on various plant and animal genome projects, this conference has been organized annually since 1992 and has become the largest gathering for genomics researchers around the world. In the first few years, the meeting focused mainly on plant genomes (PG I IV). As a regular attendee of every meeting in the last 20 years, I have witnessed how it has gradually made an impact in many areas of plant research including breeding science. As far as rice research is concerned, PAG has been the venue for disseminating the major achievements of the Rice Genome Research Program (RGP) since was it initiated in 1991. At that time, an interagency NSF/USDA/DOE delegation even came to Japan to get an overview of a major research project focusing on an agricultural crop. As a consequence, a major highlight of PG II included presentations on applications of genome mapping and analysis and the progress of genetic and physical mapping of rice and other cereal crops. Since then, genome sequencing encompassing the model plant Arabidopsis, rice and other cereal crops has progressed tremendously. And PAG serves as the venue for the progress of international collaborations involving such genome projects, and exchanging information on genome sequencing, molecular genetic analysis, bioinformatics, and applied aspects of genomics. Simultaneously, attendees have grown from a few hundreds in the first few years to more than 3,000 this year coming from over 65 countries.

In recent meetings, PAG holds about 100 academic and industry workshops reflecting the rapid advances in genome research and related technologies. From a few plants in the beginning, the workshops now focus on a wide range of plant species as genome analysis has become the foundation of plant biology. The workshops on international genome sequencing collaborations such as the International Wheat Genome Sequencing Consortium (IWGSC), Grape Genome Initiative, Lolium Genome Initiative, Sugar Cane Sequencing Initiative, Cacao Genome Sequencing Project, and Cotton Genome Initiative highlights the progress of ongoing genome sequencing efforts. Workshops with implications on practical applications of genome sequence information include genomics-assisted breeding, gene introgression, recombination mechanism, polyploidy, and speciation genomics. Other workshops address more timely topics such as bioenergy grass genomics and climate change.

Major breakthroughs in genome research as the genome sequence information became more accessible have always been a highlight in PAG meetings. A pioneering study presented this year is the precise engineering of plant genomes with sequence-specific nucleases. Highly efficient genome modifications require targeted cleavage of nucleotide sequences to generate double strand breaks and activate endogenous DNA repair pathways. In addition to the wellknown zinc finger nucleases, engineered meganucleases and transcription activator-like effector nucleases (TALENs) have been recently developed and are now widely used to pinpoint and cleave desired DNA sequences. These nucleases are highly expected to generate new types of genetically modified plants. However, whether organisms modified by these methods will be considered as GMO is still a debatable issue. There is no doubt that these new tools will have enormous impact in breeding particularly in the development of GMO crops.

Although many new advances in genomics were presented in PAG XX, it is very disappointing to note that in all workshops combined, the number of presentations given by Japanese researchers was less than 10 . Somehow, this gives an impression that Japan does not have much output in genomics recently. Of course the timing of the meeting may be considered as one factor since most researchers are busy at this time of the year. Fortunately, the first PAG-Asia will be held in Singapore on March 2013 reflecting the growing interest in genomics research in Asia. The JSPB members should use PAG-Asia as a chance to showcase our achievements in various areas genome research and particularly on how genomics has made an impact in cereal crop improvement in Japan. At the same time, we should use it as an excellent opportunity to learn the latest trends in genome research and exchange ideas with counterparts in many Asian countries.

Takuji Sasaki

Editor-in-chief

Tokyo University of Agriculture 